\title{
Calibration of planetary brightness temperature spectra at near-millimeter and submillimeter wavelengths with a Fourier-transform spectrometer
}

\author{
E. Serabyn and E. W. Weisstein
}

\begin{abstract}
A medium-resolution Fourier-transform spectrometer for ground-based observation of astronomical sources at near-millimeter and submillimeter wavelengths is described. The steps involved in measuring and calibrating astronomical spectra are elaborated. The spectrometer is well suited to planetary spectroscopy, and initial measurements of the intrinsic brightness temperature spectra of Uranus and Neptune at wavelengths of 1.0 to $1.5 \mathrm{~mm}$ are presented. ๑) 1996 Optical Society of America
\end{abstract}

\section{Introduction}

Although the Earth's atmosphere is opaque at farinfrared wavelengths, high dry sites such as the summit of Mauna Kea provide access to a number of semitransparent atmospheric windows at submillimeter and near-millimeter wavelengths ${ }^{1,2}$ [Fig. 1(a)]. As most astronomical observations in the available windows tend to be carried out with either very narrow-band heterodyne receivers ${ }^{5,6}$ or very broadband, but low-resolution, continuum bolometers, ${ }^{7,8}$ spectroscopic measurements that cover these windows in their entirety have yet to be pursued vigorously. One promising approach to the measurement of wideband astronomical spectra at these wavelengths is Fourier-transform spectroscopy, which has a proven laboratory track record in this spectral region and which has already enjoyed marked success in astronomical applications at these wavelengths with the Cosmic Background Explorer Far Infrared Absolute Spectrophotometer. ${ }^{9}$ The existence of large-aperture submillimeter-wave telescopes now provides the opportunity for extending these techniques to high spatial resolution studies of astronomical objects. ${ }^{10-14}$

Below we describe a Fourier-transform spectrometer (FTS) that we have built for astronomical obser-

The authors are with the California Institute of Technology, Pasadena, California 91125; E. Serabyn is with the Department of Physics, 320-47, and E. W. Weisstein is with the Department of Geological and Planetary Sciences, 170-25.

Received 28 August 1995; revised manuscript received 27 November 1995.

0003-6935/96/162752-12\$10.00/0

(C) 1996 Optical Society of America vations at wavelengths between 2 and $0.3 \mathrm{~mm}$ (frequencies of $150 \mathrm{GHz}$ to $1 \mathrm{THz}$ ) at the Caltech Submillimeter Observatory (CSO). This FTS has recently begun to provide broadband, moderateresolution spectra of the planets, the interstellar medium, and our own atmosphere. ${ }^{10-12}$ Here we describe the steps involved in obtaining accurately calibrated spectra with this instrument, and, in particular, we address the question of the calibration of planetary absolute brightness temperature spectra, an area in which accurate measurements at these wavelengths remain sparse.

\section{The Spectrometer}

The FTS we have constructed is an intermediateresolution scanning Michelson interferometer ${ }^{15-17}$ with a resolving power of $R=v / \Delta \nu_{\text {res }}<5000 \nu_{\mathrm{THz}}$. Here $v$ is the operating frequency, $\Delta v_{\text {res }}$ is the resolution, and $\nu_{\mathrm{THz}}$ is the frequency in terahertz. The FTS is an upgraded version of the shearing interferometer $^{17}$ used for holographic dish measurements at the CSO and remains operational in that capacity as well. As a result of the need to steer the beam in one of the interferometer's arms off axis for far-field measurements, flat end mirrors in the interferometer arms are a requirement. This excludes the use of a Martin-Pupplett configuration ${ }^{13,18}$ and forces the choice of a dielectric beam-splitter system. This choice brings a somewhat lower sensitivity but at the same time results in a much simpler optical configuration, yielding important advantages in terms of setup, ease of alignment, and reliability at a remote site. To reach the theoretical sensitivity limit, considerable effort has also been directed to understanding and eliminating systematic errors. 

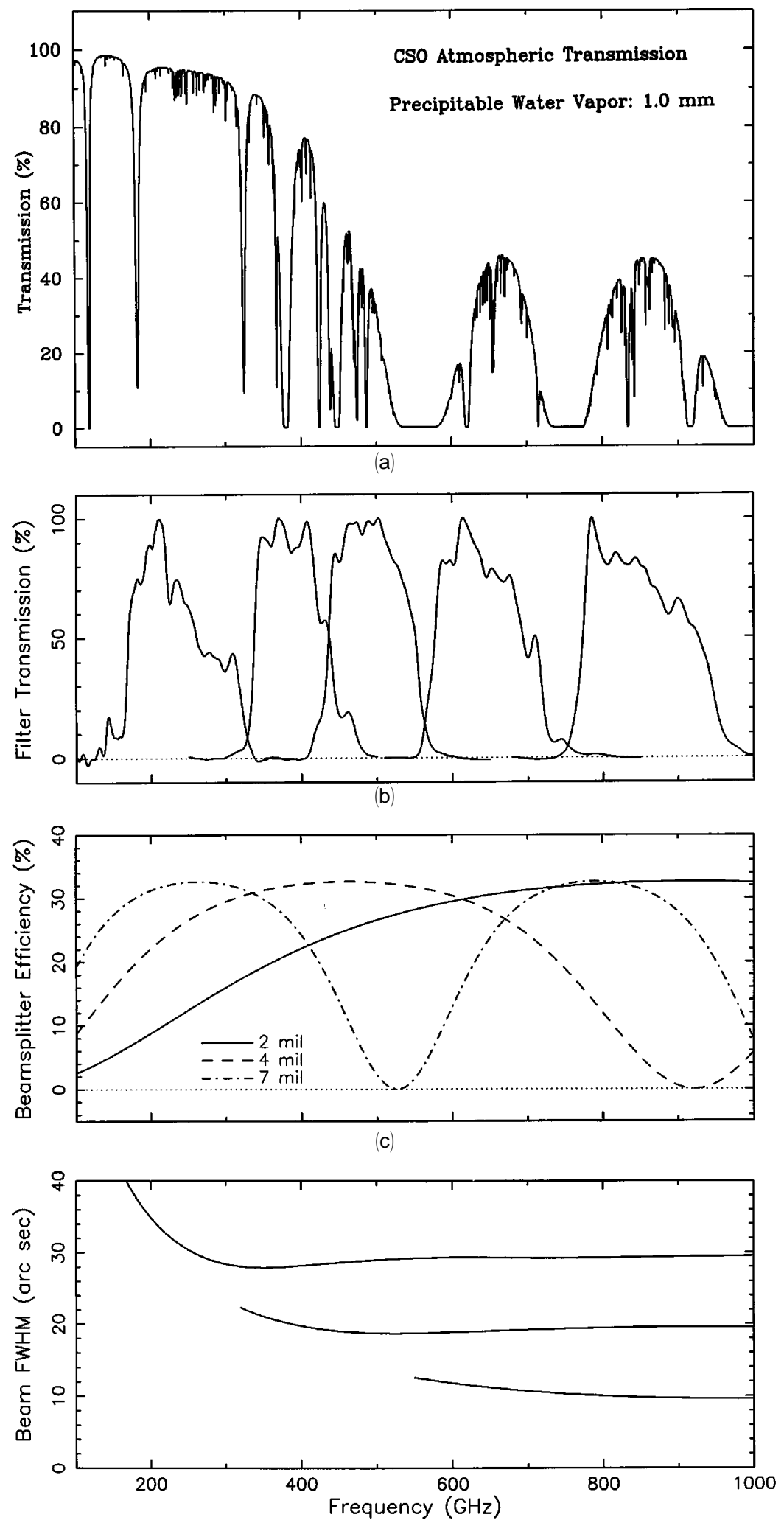

(d)

Fig. 1. (a) Model atmospheric transmission ${ }^{2}$ at the Caltech Submillimeter Observatory for 1-mm precipitable $\mathrm{H}_{2} \mathrm{O}$. (b) Normalized Fourier-transform spectrometer filter transmissions. From left to right, the approximate passband centers are at 1300, 800, 600, 450, and $350 \mu \mathrm{m}$. (c) Mylar beam-splitter efficiencies. ${ }^{3}$ The thicknesses are labeled in thousandths of an inch. (d) FWHM beam sizes calculated for the convolution of the telescope's Airy pattern with the top-hat response of an idealized Winston cone. ${ }^{4}$ From top to bottom, the nominal geometric Winston-cone fields of view are 30, 20 and 10 seconds of arc, respectively. 
The components of the FTS are affixed to a framework that is rigidly mounted at the telescope's Cassegrain focus. The optical system is open to the atmosphere, which is possible at these wavelengths only because of the low-humidity conditions prevalent on the summit of Mauna Kea. Figure 2 presents a simplified two-dimensional schematic of the current optical system. After passage through the telescope, the $F / 12.4$ beam from the secondary mirror first reflects off the two flat mirrors, M1 and M2, which are located on either side of the Cassegrain focus (Fig. 2). These mirrors serve two functions: they fold the optical path so that a collimator of appropriate focal length can fit in the available space above the telescope platform, and more importantly, they provide for a decoupling between the interferometer's internal alignment and the relative telescopeinterferometer alignment. This allows the internal FTS alignment to be set by fixed mirrors (with little or no adjustment capability), while still allowing full steering of the instrument's input beam to match to that of the telescope.

The light next hits an off-axis paraboloidal mirror, $\mathrm{P} 1$, of axial focal length $127 \mathrm{~cm}$, producing a 10-cmdiameter collimated beam and an image of the secondary (the limiting aperture) $152-\mathrm{cm}$ postreflection. The paraboloid is a numerically milled $\mathrm{Al}$ mirror that has been polished sufficiently to reflect optically (to aid in the alignment process). The root-mean-square (RMS) surface accuracy of this

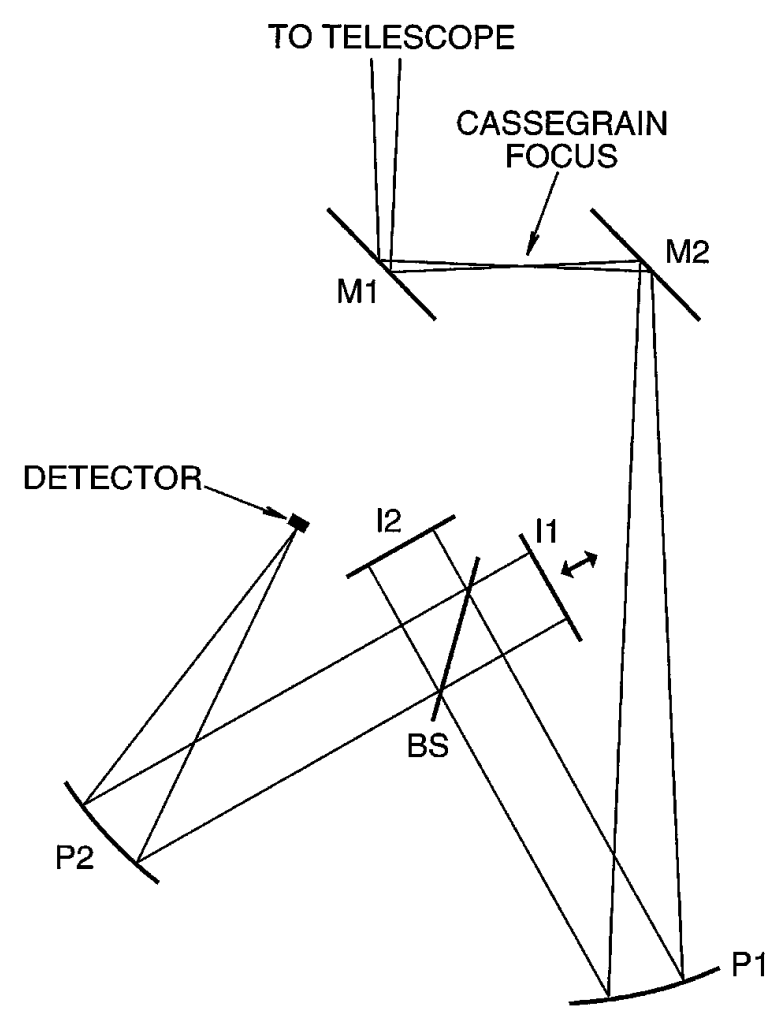

Fig. 2. Schematic layout of the FTS. M1, M2, I1, and I2 are flat front surface mirrors, P1 and P2 are off-axis paraboloids, and BS is a Mylar beam splitter. I1 translates to scan the path difference between I1 and I2. mirror is roughly $3 \mu \mathrm{m}$, or $\lambda_{\min } / 100$, where $\lambda_{\min }$, the minimum wavelength accessible through the atmosphere, is roughly $300 \mu \mathrm{m}$.

Just before the image of the secondary produced by $\mathrm{P} 1$, the beam is divided by a Mylar beam splitter, $\mathrm{BS}$, and the two resultant beams propagate to the two flat mirrors, I1 and I2. I1 is mounted on a translation stage that allows for scanning the path difference between the beams, and I2, which is coincident with one of the two equivalent secondary images, is mounted on a stepper-motor-controlled Gimbal mount (used for beam steering in holographic dish measurements ${ }^{17}$ ). I2's steering capability makes a computer-controlled grid search for its optimal tilt relative to I1 [determined by the maximization of the height of the white-light fringe (WLF)] quite straightforward. To prevent interference for radiation originating outside the beam from the secondary, an opaque Eccosorb ${ }^{19}$ aperture stop masks I2 beyond the outer diameter of the secondary image located on its surface.

After reflecting off I1 and I2, the beams are recombined at the beam splitter and then brought to an $F / 4.4$ focus by a second off-axis paraboloid, P2. The FTS uses a single ${ }^{3} \mathrm{He}$-cooled bolometer as its detecting element, which is housed in an integrating cavity at the focus of one of a number of Winstoncone light concentrators. ${ }^{4}$ To limit the background flux on the detector, five cold (liquid-He temperature) bandpass filters [Fig. 1(b)] are used to match to the available atmospheric windows. The beam-splitter thickness is also selected to optimize throughput ${ }^{3}$ [Fig. 1(c)]. The geometric acceptance angle of the Winston cones matches the $f$-number of the incoming beam, and the entrance-aperture diameters of the available cones correspond to nominal fields of view (FOV's) of 10, 20, and 30 arc sec on the sky. The shape of the observing beam results from the convolution of the telescope's focal-plane Airy pattern with the Winston-cone top-hat geometric response function. This convolution can be carried out numerically, and Fig. 1(d) shows the resultant beam FWHM's for the three available cones. For most frequencies above the cones' diffraction cutoffs, the resultant beam sizes are relatively constant.

The translation stage has a full travel of $50 \mathrm{~cm}(\mathrm{a}$ length dictated by space constraints) and is driven by means of a precision lead screw and dc motor. To minimize instrumental distortions as the telescope tips over in zenith angle, the optical system was designed so that the axis of the translation stage is oriented parallel to the telescope's elevation axis, and at a midrange zenith angle of $50^{\circ}$ the translation stage is at its optimum, completely horizontal orientation. The last $0.75 \mathrm{~cm}$ or so at each end of the stage's travel are not used in scanning, to avoid binding near the bearings and to allow a safe stopping margin. The stationary mirror is normally positioned so that the equal path, or WLF, position occurs with the translating mirror roughly $2.5 \mathrm{~cm}$ from its beam-splitter-side terminus. The maxi- 
mum one-sided travel $L_{1}$ is then $46 \mathrm{~cm}$. Taking the unapodized spectral resolution as the half-width of the sinc function resulting from cosine transforming the one-sided window function, ${ }^{16}$ or

$$
\Delta v_{\text {res }}=1.207 \frac{c}{4 L_{1}},
$$

where $c$ is the speed of light, yields a finest unapodized spectral resolution of $0.197 \mathrm{GHz}$. The optical configuration also allows for symmetric scans of up to $5 \mathrm{~cm}$ in total travel (so that $L_{1}=2.5 \mathrm{~cm}$ in the above formula), yielding a two-sided spectral resolution of $3.6 \mathrm{GHz}$. The stationary mirror can also be remounted $2 \mathrm{~cm}$ farther from the beam splitter, yielding the finest two-sided resolution of $2.0 \mathrm{GHz}$. For short scans, little difference is seen between one-sided and two-sided transforms, although the two-sided transforms provide phase information useful in assessing data quality (Section 4).

Position along the scan direction is determined by a linear optical encoder mounted on the translation stage. Because of the long wavelengths involved, the accuracy of an encoder system is adequate, and no He-Ne interferometer is employed. The linear encoder has two channels, one of which provides pulses spaced at $20-\mu \mathrm{m}$ intervals (i.e., $\lambda_{\min } / 15$ ), which are used to trigger sampling of the bolometer signal by an analog-to-digital converter, while the second channel supplies a single pulse [the reference pulse $(\mathrm{RP})]$ at a fixed location near the WLF position. Circuitry is also in place to convert the trigger pulses to 10 - $\mu \mathrm{m}$ spacing for the more rapidly modulated interferograms. With 20 - $\mu \mathrm{m}$ sampling, the submillimeter interferogram fringes are sampled above the Nyquist rate for all frequencies up to $3.75 \mathrm{THz}$. Subterahertz signals are thus quite liberally oversampled, bringing advantages both in locating the WLF accurately and in the recognition of interferogram asymmetries. Both of these aspects are useful in eliminating residual phase errors, with the result that one-sided transforms need no phase correction beyond that required for off-center sampling (Section 3).

The interferometer is operated in rapid-scan mode, producing modulated signals at electrical frequencies $f=2 v / \lambda$, where $v$ is the stage velocity and $\lambda$ is the wavelength of the radiation. The optimal mirror speed depends on filter passband and varies from roughly $0.9 \mathrm{~cm} \mathrm{~s}^{-1}$ for the lowest frequency passband to $0.45 \mathrm{~cm} \mathrm{~s}^{-1}$ for the highest, resulting in electrical fringes in the $6-30-\mathrm{Hz}$ range. This band was selected to avoid sky-fluctuation noise on the low side (which typically occurs at $\leqslant 1 \mathrm{~Hz}$ in clear weather and $<5 \mathrm{~Hz}$ in marginal weather) and microphonic $(>30$ $\mathrm{Hz})$ and line $(60 \mathrm{~Hz})$ pickup on the high side. The electrical bandwidth is defined by a high-pass RC filter in the bolometer preamp with a 3-dB cuton of $0.2 \mathrm{~Hz}$ and a six-pole low-pass Bessel filter (for maximally flat phase response) with a cutoff of $80 \mathrm{~Hz}$. The Bessel filter prevents the aliasing of high- frequency noise into the passbands defined by the submillimeter filters, and its cutoff could safely be lowered somewhat, but as all noise between 30 and $80 \mathrm{~Hz}$ is out of the range of interest, this band is retained for diagnostic purposes. Figure 3 shows that the extra (primarily microphonic) noise present in the $30-80-\mathrm{Hz}$ band can be removed post facto, even for weak signals that require the summation of multiple scans, by first transforming the raw interferograms to spectral space, zeroing all spectral components outside the optical filter cutoffs, and then inverse transforming back to interferogram space. Thus the interferograms themselves remain uncorrupted by out-of-band noise.

Within the submillimeter passbands of interest, sky background limited noise performance is found at the higher frequencies, while at lower frequencies, reduced background levels lead to the detector noise limit being reached first. After a total observation time of $t$ seconds, spectra show a typical RMS noise in antenna temperature units (Section 4) of $\sigma \approx$ $2\left(\Delta v_{\text {res }} \sqrt{t}\right)^{-1}$, with $\Delta v_{\text {res }}$ in gigahertz. ${ }^{11}$
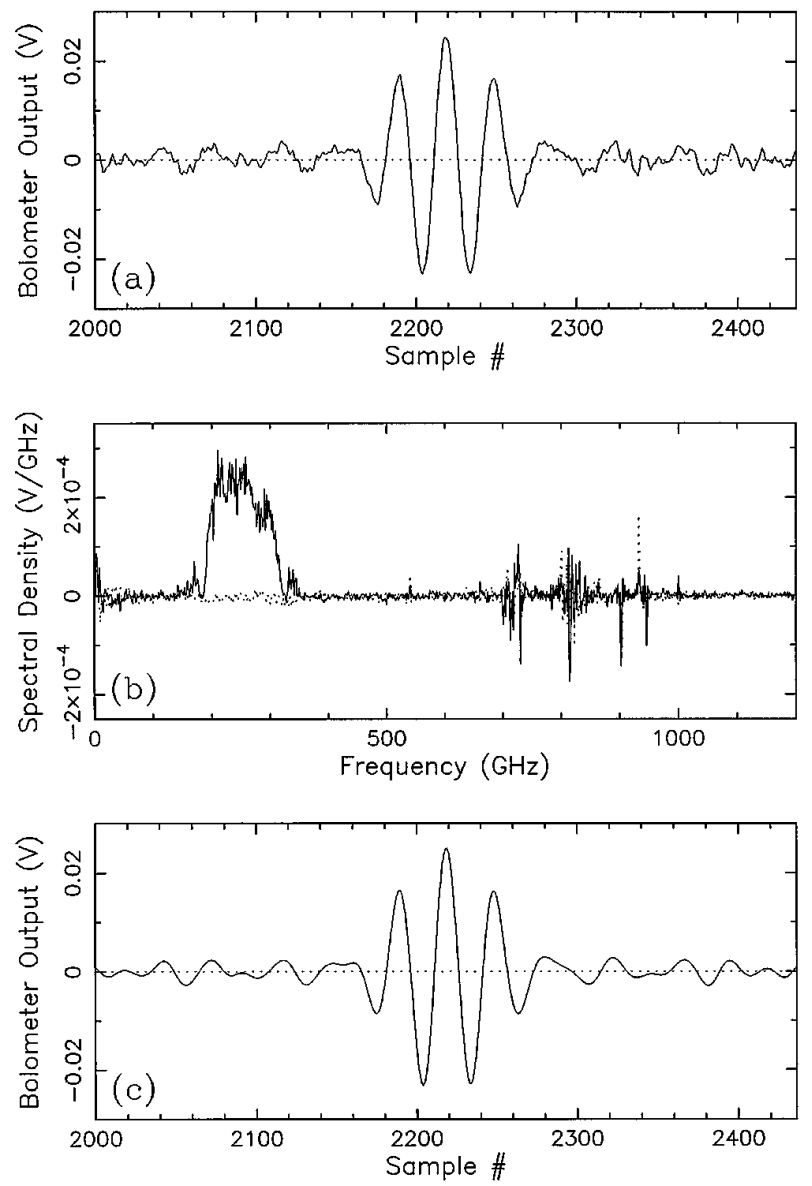

Fig. 3. (a) Observed interferogram for Mars (i.e., on-off) resulting from the summation of 15 scans of mirror I1 (all in the same direction). (b) Fourier transform of (a), with the solid (dotted) curve showing the real (imaginary) part of the transform. Microphonic pickup is evident from 700 to $1000 \mathrm{GHz}$. (c) Interferogram resulting from zeroing spectrum from 0 to $100 \mathrm{GHz}$ and above $500 \mathrm{GHz}$, and then transforming back to interferogram space.

1 June 1996 / Vol. 35, No. 16 / APPLIED OPTICS

2755 


\section{Scan Registration and Quality Control}

For astronomical sources, data reduction and calibration require several steps beyond simple Fourier transformation of interferograms: (a) coalignment of different scans, (b) registration of interferograms from scans in the two directions of mirror motion, (c) correction for off-center sampling of interferograms, (d) identification and exclusion of bad scans, (e) correction for emission in the interferometer's second input port, (f) correction for atmospheric absorption and hot spillover, and $(\mathrm{g})$ correction for the coupling of the source to the telescope beam. The first four of these are discussed only briefly in the following paragraphs, as they are more-or-less standard issues in Fourier-transform spectroscopy, whereas the latter three are discussed at length in the succeeding sections.

For weak sources, many scans may need to be coadded to achieve an adequate signal-to-noise ratio in the final spectrum. However, the weakness of source interferograms prevents their direct use in finding WLF positions. Thus, to coalign multiple interferograms, the displacement between the WLF position and the RP position is first determined from observations of an ambient temperature blackbody source (Eccosorb) that fills the beam (reference scans in the following). Of course, this WLF - RP difference need not be an integer, and a quadratic polynomial is typically fitted to the central five points of the reference interferogram (having scanned past the WLF to acquire the needed data) to determine the nonintegral part of the WLF - RP difference. Individual on- and off-source scans in a given direction are registered relative to each other by shifting all scans so that their RP's coincide in sample number, thus correcting for slight drifts in scan start location. The nearest integer to the measured WLF - RP difference is then used to shift to the nominal WLF position. The interferograms can then be summed and transformed and the nonintegral part of the WLF - RP difference used to correct the transformed spectra for off-center sampling errors by the application of the standard multiplicative phase correction. ${ }^{15,16}$ Because of the intrinsic symmetry of the measured interferograms, no higherorder corrections such as dechirping ${ }^{15}$ are necessary. Of course all these shifts and phase factors must be determined separately for scans in opposite directions, as phase delays in the amplifier circuitry lead to differing time delays in the bolometer signal (and hence in the measured WLF position) for the two directions. Thus scans in opposite directions are usually combined only in spectral space.

As the same complex phase correction (which derived from the average of the reference scans) is typically applied to all nonreference scans and the calibration procedure outlined in Section 4 involves a ratio of spectra, this phase factor actually cancels out in the simplest treatment of the data. However, both the location of the $\mathrm{RP}$ relative to the start of the scan and the WLF - RP difference show a slow drift with time, requiring a slightly more careful treatment of phase errors for lengthy observations. While tracking a source, the WLF - RP displacement is typically stable to better than $1 \mu \mathrm{m}$, or $\lambda_{\min } / 300$, for periods of roughly $20-30 \mathrm{~min}$, a stability more than adequate for typical on-off observation cycles. However, during the longer time scale of a full source transit, thermal expansion and zenithangle-dependent instrumental flexure limit the stability to $\pm 3 \mu \mathrm{m}$, and so the WLF - RP difference is usually remeasured on a 20-30-min time scale. When a simple polynomial is fitted to the WLF - RP differences determined for the resulting set of reference scans, it becomes possible to phase correct all on- and off-source scans for changing conditions. As each scan then requires a slightly different phase correction, the scans must be Fourier transformed individually before phase correcting and summing in spectral space.

Although these procedures are sufficient for most scans, occasionally the bolometer signal can be corrupted by one of several mechanisms: voltage spikes ( 1 or 2 samples wide) in the bolometer signal (caused by cosmic-ray hits on the detector or static discharge between the unsuspecting operator and the control keyboard), extraneous pulses in the trigger line (causing extra samples to be taken), and rapid sky variations. The narrow spikes are the easiest to correct for, as these can be detected in oversampled interferograms by their anomalously large signal changes compared with nearby gradients. If not removed, they lead to a ripple in the transformed spectra. Once spikes are detected by search software, the anomalous bolometer readings are replaced by an interpolation through the neighboring clean channels. Occasional extraneous trigger pulses, most likely caused by electronic pickup in the noisy telescope environment, shift the remainder of the interferogram by one sample, and can be detected by a comparison of all scans to a selected template scan (assumed uncontaminated). The RMS difference of subsequent scans from this template can be calculated and plotted versus scan number, and deviations from the norm that are due to a midinterferogram shift are made manifest by virtue of their anomalously large RMS's. The offending scans can then either be rejected (if many scans are available) or corrected (if few are in hand) by the location and the removal of the doubled voltage sample. This template comparison also provides a means of assessing the magnitude of sky variations, as air mass and weather variations also serve to alter the individual interferograms noticeably (Fig. 4). In addition to secular RMS changes in sky interferograms, another diagnostic for subpar weather conditions is a deviation between RMS's for the two scan directions (calculated separately). Agreement between results for both scan directions is of course a necessary criterion for data acceptance. 

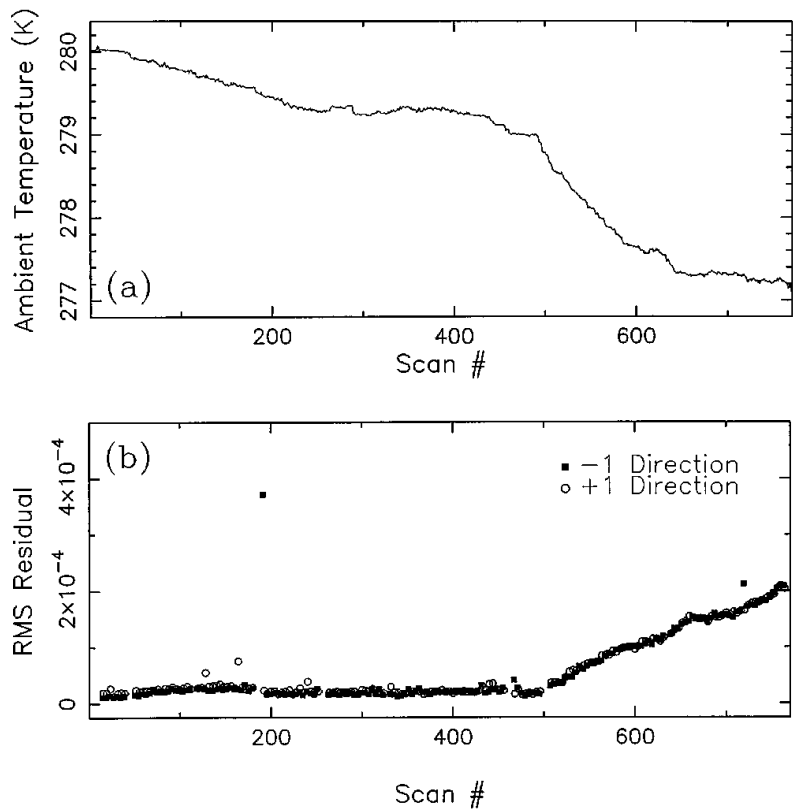

Fig. 4. (a) Plot of the measured ground-level atmospheric temperature versus FTS scan number on 19 September 1995. (b) Plot of the RMS difference of succeeding blank sky interferograms relative to the first. The two scan directions are plotted with empty circles and solid squares, respectively. Note the rise in RMS beginning near scan 500, which corresponds to the drop in ambient temperature seen in panel (a). Note also the obvious deviations of the few bad scans (e.g., $\approx 190,720)$ from the rest.

\section{Corrections for Ambient Emission}

Michelson interferometers pass radiation from two different entrance ports to the detector. ${ }^{16,18}$ In our case, the first input port corresponds to radiation from the telescope, and the second input port refers to light that arrives at the beam splitter from the detector side. The emission from the detector region that falls into the interferometer beam is primarily ambient temperature emission (the cold detector does not emit much, but its surroundings do) and so does not vary significantly on short time scales. This emission can then be subtracted from on-source observations at the same time that emission from the sky is subtracted. Thus it is natural to treat these two topics together. To subtract ambient and port 2 emission from scans on an astronomical source, each pair of on-source scans (one in each direction of mirror motion) is followed immediately by a pair of scans off the source position on blank sky at a similar air mass. The duration of a pair of scans (100-200 s for full resolution) is sufficiently long that, for the off-scan pair, the telescope is offset back to the position the source had occupied at the beginning of its scans, with tracking resuming there. Of course this procedure requires weather and instrumental conditions to remain stable on the time scale of four scans. The on-off subtraction is followed by Fourier transformation to spectral space, but as in practice the order of operations (differencing, then transformation versus transformation, then differencing) does not yield noticeable differences in the final spectra, we proceed below as if all scans are immediately transformed to spectral space, and all operations occur there.

It is possible to calibrate FTS observations of astronomical sources in a manner similar to that typically employed for calibration of heterodyne observations at near-millimeter wavelengths, but with the parameters now treated as functions of frequency. Before we describe the procedure, it is important to note that the relevant instrumental beam is not simply the beam emerging (considering the transmitting case) from the Winston cone, which unfortunately has sidelobes beyond the geometric acceptance angle. As only interferometrically modulated flux is detected by the ac-coupled bolometer circuitry, the angular response of the Winston cone can be modified in the interferometer by a suitably placed aperture stop, and it is to this end that an opaque mask obscures mirror I2's surface beyond the outer diameter of the secondary image located there (Section 2). Rays outside of the unobstructed aperture then cannot interfere, which effectively removes the Winston-cone sidelobe response completely. The result is that a much more sharp-edged interferometrically modulated beam passes through the interferometer to couple to the telescope secondary with high efficiency. Of this beam, a fraction $\eta_{\text {sky }}(\nu)$ reaches the sky (hereafter the sky beam), and $1-\eta_{\text {sky }}(v)$ terminates on ambient temperature surfaces such as those behind the central hole in the primary and the panel gaps, as well as on the feedlegs (collectively called the hot spillover). In practice, the coupling efficiency of the sky beam to astronomical sources was found to increase by just under a factor of 2 with the aperture stop in place.

The equations of radiative transfer and antenna theory, together with the inclusion of port 2 emission, then lead to the following equation for the blank sky (off) voltage spectrum ${ }^{18,20-25}$ :

$$
\begin{aligned}
V_{\text {off }}(v)= & G_{v}\left\{\left[1-\exp \left(-\tau_{v}\right)\right] \eta_{\text {sky }}(\nu) B_{v}\left(T_{\text {sky }}\right)\right. \\
& \left.+\left[1-\eta_{\text {sky }}(\nu)\right] B_{v}\left(T_{\text {hot }}\right)-\sum_{i} \eta_{2, i} B_{v}\left(T_{2, i}\right)\right\},
\end{aligned}
$$

where $G_{v}$ is a combination of optical-transmission and electrical-gain factors and $\tau_{\nu}$ is the atmospheric optical depth at frequency $\nu$. The various $B_{v}$ 's are blackbody-specific intensity functions, the $\eta_{2, i}$ 's are the coupling efficiencies to surfaces of various temperatures in the port 2 beam (subject to $\Sigma_{i} \eta_{2, i}=1$ ), and the various $T$ 's are physical temperatures: $T_{\text {sky }}$ of the sky, $T_{\text {hot }}$ of ambient temperature surfaces, and the $T_{2, i}$ 's of all the surfaces subtended by the port 2 beam including $T_{\text {hot }}, 77 \mathrm{~K}, 4 \mathrm{~K}$, and $0.3 \mathrm{~K}$ (the latter three refer to radiation shield and detector temperatures). All the $\eta_{2, i}$ 's are slow functions of frequency, but this dependence is not displayed explicitly. 
Finally, the sign of the last term is negative because the modulation of port 2 radiation occurs with sign ${ }^{18}$ opposite to that of port 1.

The on-source spectrum contains all the terms present in Eq. (2), in addition to the signal from the source. The on-off difference spectrum is then due solely to the source term, given by

$$
V_{\text {on }}(\nu)-V_{\text {off }}(v)=G_{v} \eta_{\text {sky }}(\nu) \exp \left(-\tau_{v}\right) \int I_{v}^{\mathrm{sou}}(\Omega) P_{v}(\Omega) \mathrm{d} \Omega,
$$

where $I_{v}^{\mathrm{sou}}(\Omega)$ is the specific intensity distribution of the source as a function of angular coordinates (represented by $\Omega$ ), and $P_{v}(\Omega)$ is the normalized power pattern of the sky beam at frequency $\nu$. Although the difference spectrum thus removes atmospheric, hot spillover, and port 2 emission, it does not correct for atmospheric absorption, and it retains a dependence on the gain and the sky-efficiency factors. However, both of these factors can be eliminated with the aid of the spectrum of an ambient temperature load. ${ }^{21}$ To this end, a pair of FTS scans is also taken of an ambient temperature Eccosorb sheet that completely fills input port 1 . (These scans also serve as the reference scans used in deriving the WLF position; Section 3.) The observed spectrum of the ambient (or hot) load is

$$
\begin{aligned}
V_{\text {hot }}(v)= & G_{v} \mid \eta_{\text {sky }}(v) B_{v}\left(T_{\text {hot }}\right)+\left[1-\eta_{\text {sky }}(v)\right] B_{v}\left(T_{\text {hot }}\right) \\
& \left.-\sum_{i} \eta_{2, i} B_{v}\left(T_{2, i}\right)\right\} .
\end{aligned}
$$

Taking the difference between this signal and the off spectrum leaves

$$
\begin{aligned}
V_{\text {hot }}(\nu)-V_{\text {off }}(v)= & G_{v} \eta_{\text {sky }}(\nu)\left\{B_{v}\left(T_{\text {hot }}\right)\right. \\
& \left.-\left[1-\exp \left(-\tau_{v}\right)\right] B_{v}\left(T_{\text {sky }}\right)\right] .
\end{aligned}
$$

If we now assume that the sky and the ambient surfaces are at the same temperature, it follows that

$$
V_{\text {hot }}(v)-V_{\text {off }}(v)=G_{v} \eta_{\text {sky }}(v) \exp \left(-\tau_{v}\right) B_{v}\left(T_{\text {hot }}\right) .
$$

Although this assumption is valid for absorption by low-altitude water vapor, it cannot correct properly for absorption by high-altitude ozone (which is at a lower temperature). However, the narrow $\mathrm{O}_{3}$ lines are less important, as they affect only specific frequencies. Equations (3) and (6) contain identical prefactors, and so a simple ratio yields

$$
\int I_{v}^{\mathrm{sou}}(\Omega) P_{v}(\Omega) \mathrm{d} \Omega=B_{v}\left(T_{\mathrm{hot}}\left[\frac{V_{\text {on }}(v)-V_{\text {off }}(v)}{V_{\text {hot }}(v)-V_{\text {off }}(v)}\right] .\right.
$$

The left-hand side of this equation is simply the coupling of the source flux to the telescope's sky beam. At long wavelengths (in or near the Rayleigh-
Jeans limit), this equation can be converted to an equivalent one in terms of antenna temperatures. First we introduce the definition of the RayleighJeans source brightness temperature:

$$
T_{B}^{\mathrm{sou}}(\Omega) \equiv \frac{I_{v}^{\mathrm{sou}}(\Omega) \lambda^{2}}{2 k},
$$

where $k$ is Boltzmann's constant. Note that this is not a Rayleigh-Jeans approximation; with this definition, the true source flux can be recovered from $T_{B}^{\mathrm{sou}}(\Omega)$ by inversion of the equation. Now, with the definition of antenna temperature,${ }^{20}$ or, as is more appropriate for our case, antenna temperature corrected for atmospheric absorption and hot spill$\operatorname{over}^{21,22}$ (and port 2 emission) $T_{A}^{*}(v)$, which is given by

$$
T_{A}^{*}(v) \equiv \int T_{B}^{\mathrm{sou}}(\Omega) P_{v}(\Omega) \mathrm{d} \Omega,
$$

the combination of Eqs. (7)-(9) then yields

$$
T_{A}^{*}(v)=\frac{\lambda^{2} B_{v}\left(T_{\mathrm{hot}}\right)}{2 k}\left[\frac{V_{\text {on }}(v)-V_{\text {off }}(v)}{V_{\text {hot }}(v)-V_{\text {off }}(v)}\right] .
$$

When the full Planck expression for the ambient temperature blackbody-specific intensity is inserted, the final calibrated spectrum in antenna temperature units (in degrees Kelvin) is

$$
T_{A}^{*}(v)=\frac{h v / k}{\exp \left(h v / k T_{\text {hot }}\right)-1}\left[\frac{V_{\text {on }}(v)-V_{\text {off }}(v)}{V_{\text {hot }}(v)-V_{\text {off }}(v)}\right],
$$

where $h$ is Planck's constant. In the RayleighJeans limit, Eq. (11) reduces to

$$
T_{A}^{*}(v)=T_{\text {hot }}\left[\frac{V_{\text {on }}(v)-V_{\text {off }}(v)}{V_{\text {hot }}(v)-V_{\text {off }}(v)}\right],
$$

Figure 5 graphically summarizes this calibration procedure for the case of two-sided interferograms, for which the phase of the complex Fourier transform is available for inspection. Figures 5(a), 5(b), and 5(c) show raw emission spectra of blank sky (off) with two strong atmospheric $\mathrm{H}_{2} \mathrm{O}$ emission lines evident at 183 and $325 \mathrm{GHz},{ }^{26}$ Mars (i.e., Mars-off), and hot-off (i.e., ambient temperature Eccosorb-off), respectively. Note that the channel-to-channel variations are similar in Figs. 5(b) and 5(c) and reflect passband features that should divide out. At higher resolution these features are seen to be a coherent ripple caused by resonances in the Winston-cone transmission function. ${ }^{10}$ Next, Fig. $5(\mathrm{~d})$ shows the antenna temperature spectrum of Mars, the result of rationing Figs. 5(b) and 5(c) and scaling as in Eq. (11). The central 100 or so gigahertz of the passband show a relatively quiet spectrum in which the passband features have indeed largely divided out. Of course, near and beyond the edges of the filter and atmo- 

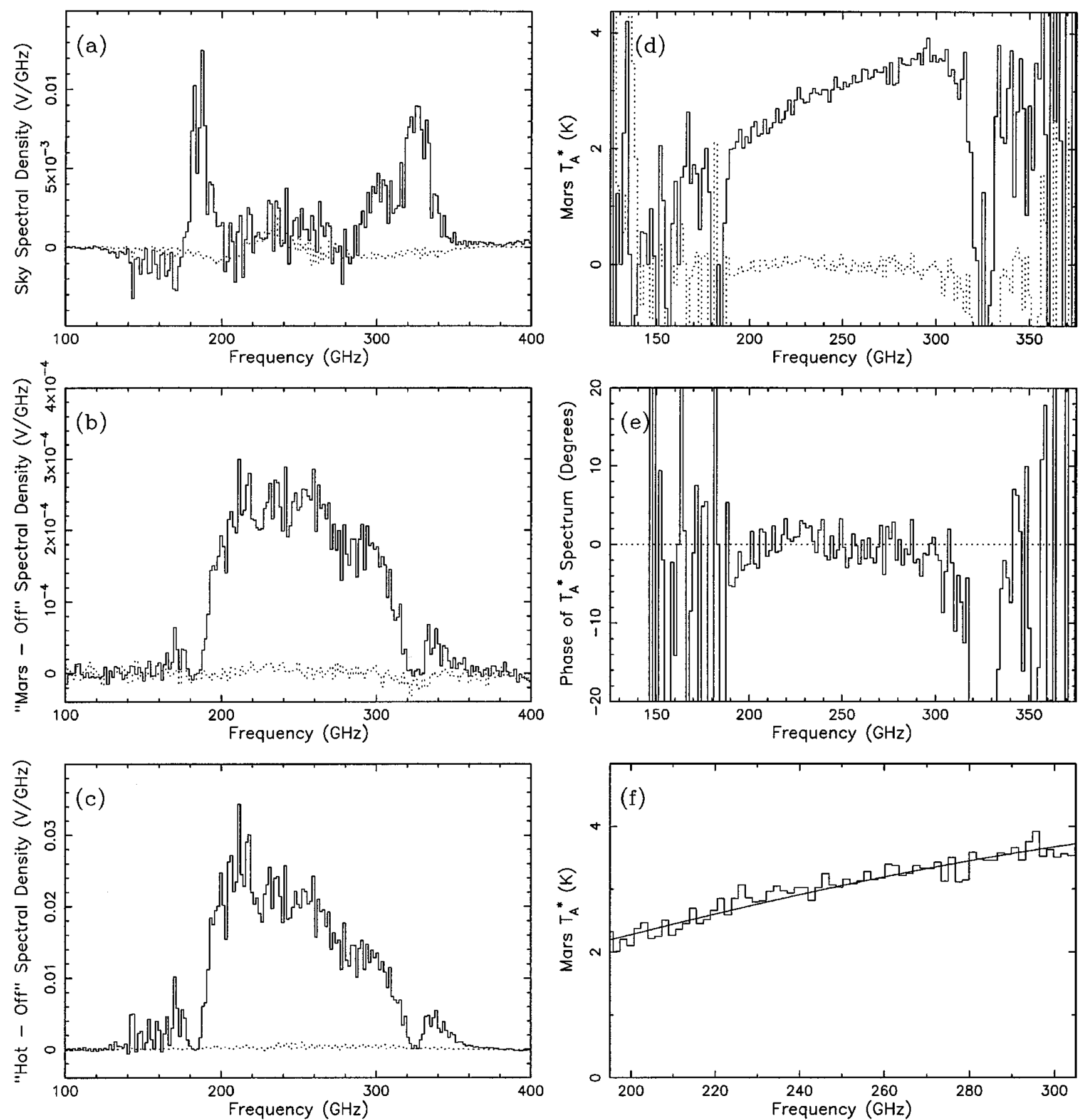

Fig. 5. (a) Sky emission spectrum (off) observed through the 1300- $\mathrm{mm}$ filter. The solid curve shows the real part and the dotted curve shows the imaginary part of the two-sided Fourier transform [also in (b)-(d)). (b) Observed Mars-off spectrum. (c) Observed hot-off spectrum. (d) The spectrum of Mars in antenna temperature, $T_{A}^{*}$, units (degrees Kelvin) the result of dividing panels (b) and (c), and scaling by means of Eq. (11). (e) Phase of the complex spectrum seen in panel (d). The dotted line in this case is a zero line, for reference. (f) A blowup of the good part of the panel (d) spectrum (histogram), and a beam-coupling model fit to the data ( solid curve), with $\eta_{\operatorname{moon}}=0.83$, $\epsilon=15 \mu \mathrm{m}$, and $\mathrm{FOV}=28$ arc sec.

spheric passbands, the noise level rises dramatically as the transmission declines. The phase of this final spectrum, shown in Fig. 5(e), is quite close to zero $\left(<3^{\circ}\right)$ across most of the filter passband, attesting to the quality of the data. However, beyond the $195-305-\mathrm{GHz}$ range, the phase deviates from zero, implying that data beyond that range are suspect. For clarity, the high-quality section of Mars' calibrated antenna temperature spectrum is shown in Fig. $5(\mathrm{f})$.

\section{Source Coupling}

Although this calibration procedure puts our spectra on an antenna temperature scale analogous to that of most millimeter- and submillimeter-wavelength heterodyne observations, this scale is unfortunately dependent on both telescope aperture and illumination through the $P_{v}(\Omega)$ factor in Eq. (9). This can be seen clearly in Fig. 5(f), in which Mars' observed antenna temperature rises rapidly with frequency as 
the telescope beam narrows. The final issue to address is thus the conversion from the measured antenna temperature spectrum to the source-intrinsic brightness temperature spectrum, $T_{B}^{\mathrm{sou}}(\nu)$. As this requires inversion of Eq. (9), a priori source structure information is usually necessary. In the case of the planets, this information is available, and so we now focus on this case.

We assume below that the intrinsic brightness temperature of a given planet can be represented by an elliptical disk of uniform temperature $T_{p}(v)$ (thus ignoring limb darkening), which is a function of frequency. Then Eq. (9) reduces to

$$
T_{p}(v)=\frac{T_{A}^{*}(v)}{\eta_{c}(v)},
$$

where $\eta_{c}(\nu)$, the source coupling efficiency, is given by the fraction of the telescope beam subtended by the planetary disk;

$$
\eta_{c}(v)=\int_{\text {planet }} P_{\nu}(\Omega) \mathrm{d} \Omega
$$

The value of this integral is of course determined entirely by the properties of the telescope beam, which in general consist of three components: a narrow main beam, which results from diffraction by the full aperture, a broader and much weaker error pattern, which is caused by panel misalignment and surface imperfections, and a wide-angle component, which is due primarily to beam spillover past the secondary ${ }^{20-25}$ (note that atmospheric seeing fluctuations are unimportant at these long wavelengths). Conversion from $T_{A}^{*}(v)$ to $T_{p}(v)$ is thus in general nontrivial, even for simple disklike sources. However, there are two ways in which this conversion can be accomplished. First, measurement of the telescope-beam parameters can be used to establish a model for $P_{\nu}(\Omega)$, which can then be integrated over the solid angle that corresponds to the size of the planetary disk in question [Eq. (14)]. Second, when a single, well-characterized planetary source is used as an absolute calibrator, the remaining planets can be calibrated in relative fashion. ${ }^{27,28}$ In the remainder of this section, we outline both approaches and their applications and present initial results obtained with each in our lowest-frequency filter.

\section{A. Beam Modeling}

The construction of a beam model is particularly profitable in our context for two reasons. First, because of the beam-width variation across the observed band, the broadband spectrum provided by a FTS contains an abundance of beam-profile information, and even a single observation of a lone planet provides as many beam-shape constraints as frequencies measured. Second, at our high frequencies (compared with radio and microwave frequencies), the widths of both the main beam $\left(\theta_{\mathrm{mb}} \approx \lambda / D\right.$, where $D$ is the primary diameter) and the error beam $\left(\theta_{\text {error }} \approx \lambda / P\right.$, where $P$ is a typical panel diameter $)$ are small. Both these beam components thus fall completely within the angular diameter of an extended source as large as the Moon, allowing the integral over both beam components to be obtained quite simply from a FTS spectrum of the full Moon. The ratio [Eq. (13)] of the full Moon's antenna temperature to its maximum physical temperature when full, ${ }^{29,30}$ $390 \mathrm{~K}$, then provides $\eta_{c}(v)$ for the Moon, hereafter $\eta_{\text {moon }}(v)$, which is the desired integral of $P_{v}(\Omega)$ over both the main and the error beams. Thus division of a planetary spectrum by that of the Moon (both already in $T_{A}^{*}$ units) corrects for the efficiency loss that is due to the wide-angle contribution, leaving only the coupling to the main and the error beams to consider.

We next use the fact that the main- and the error-beam components are not independent: the power lost from the main beam because of surface irregularities appears in the error pattern, with the fraction in the main beam given by ${ }^{31,32} \exp \left[-(4 \pi \epsilon \nu / c)^{2}\right]$, where $\epsilon$ is the RMS telescope surface error. Because the planets' angular diameters are typically comparable with (or, for Uranus and Neptune, smaller than) the CSO's main-beam size, the main beam dominates the coupling to most of the planets (excluding the case of large planets at high frequencies), and so the details of the error pattern other than its integral, $\eta_{\text {moon }}(v)\left\{1-\exp \left[-(4 \pi \epsilon v / c)^{2}\right]\right\}$, are not important. Thus the simplest applicable telescope-beam model is that of a diffraction-defined main beam (of Airy, Gaussian, or related shape) of integrated power $\eta_{\operatorname{moon}}(v) \exp \left[-(4 \pi \epsilon v / c)^{2}\right]$, which depends on only the two measurable quantities $\eta_{\text {moon }}(v)$ and $\epsilon$ (in addition to those that specify the main beam shape). At long wavelengths, the surface RMS factor approaches unity and becomes unimportant. To complete the model, the telescope beam must further be convolved with the Winston-cone top-hat response function, which introduces a final parameter, the Winstoncone FOV. Finally, integrating the resultant beam over a disk the angular size of the planet in question yields the net source coupling [Eq. (14)].

Work along these lines has begun, but poor weather has thus far limited our observations of the Moon. The data in hand from earlier observation runs (with instrumental setups somewhat different from those used for the planetary observations discussed below) imply a Moon coupling efficiency of roughly 0.8 . The accuracy of this estimate $( \pm 0.03)$ is as yet insufficient to calibrate accurately planetary spectra relative to the Moon, but the Moon's high signal strength should enable the determination of $\eta_{\text {moon }}(\nu)$ to roughly $1 \%$ with future observations. Given the remaining uncertainties in both this parameter and in the nominal geometric FOV's of the Winston cones, for the time being we take the approach of simply assessing the beam-coupling procedure, with a view to estimating the relevant parameters for 
later refinement. To this end, we have carried out the beam-coupling calculations described above and applied them to observations of Mars acquired at the CSO on 22 July 1995.

Given an assumed intrinsic brightness temperature spectrum for Mars and a beam-model calculation for $\eta_{c}(v)$, Eq. (13) yields a prediction for the antenna temperature expected from the planet (as a function of the model parameters listed). To illustrate, Fig. 5(f) includes a predicted $T_{A}^{*}$ spectrum for Mars (the solid curve) superposed on the observed spectrum, for an Airy main-beam shape, and for the parameters $\eta_{\text {moon }}(v)=0.83$ (independent of $v$, as indicated by our partial measurements), $\epsilon=15 \mu \mathrm{m}$ (as derived from holographic measurements ${ }^{12}$ ), and FOV $=28$ arc sec. The assumed Mars brightness temperature spectrum is based on the Rudy ${ }^{33}$ model. Leaving aside the details of this model, we note here only that the model is based on centimeter-wave observations and allows for a range of parameters whose values are uncertain at submillimeter wavelengths. We used midrange values in the model: a dielectric constant of 2.5 and an absorption length of $15 \lambda$. As Fig. $5(\mathrm{f})$ shows, this beam model is quite capable of reproducing the strength and the shape of Mars' observed spectrum with only slight deviations of $\eta_{\text {moon }}(v)$ and FOV from their nominal values of 0.8 and 30, respectively. These slight modifications are well within the accuracy with which these quantities are currently known, but this situation should improve with further observations. Thus the good fit to Mars' observed antenna temperature spectrum provides confidence that, with more complete beam calibrations, the modeling procedure can be successfully inverted to yield a calibrated submillimeter brightness temperature spectrum for Mars, based on a comparison of its antenna temperature spectrum with that of the Moon.

\section{B. Relative Calibration}

Although Subsection 5.A. presented a means for calibrating a planet against the Moon, the more standard approach is to calibrate the majority of the planets against a primary reference planet. In terms of well-characterized primary flux calibrators, Mars is the only feasible planetary candidate, but until the direct submillimeter-wavelength calibration proposed in Subsection 5.A. is completed, either centimeter-wave or infrared measurements must be extrapolated to the submillimeter region. Then, with the resultant known Mars temperature, a second planet can be relatively calibrated if the $\eta_{c}(v)$ 's for the two planets are known or calculated. The brightness temperature of the second planet is then given by

$$
T_{p 2}(v)=T_{p 1}(v) \frac{T_{A}^{*}(2)}{T_{A}^{*}(1)} \frac{\eta_{c}(1)}{\eta_{c}(2)}
$$

The beam-coupling ratio in Eq. (15) can either be calculated from the beam model, or better yet, set to unity if the planets under consideration have similar angular sizes on the date of observation. ${ }^{10}$ Note that, in this relative approach, the Moon efficiency divides out, and the detailed shape of the main beam becomes less important. In particular, if both the primary and the secondary planets are much smaller than the main beam, the beam-coupling ratio reduces to the ratio of the solid angles, $\Omega(1)$ and $\Omega(2)$, that the two planets subtend, so that

$$
T_{p 2}(v)=T_{p 1}(v) \frac{T_{A}^{*}(2)}{T_{A}^{*}(1)} \frac{\Omega(1)}{\Omega(2)} .
$$

Using this relative calibration approach, we have now acquired and calibrated spectra of Uranus and Neptune covering the $1.0-1.5-\mathrm{mm}$ range. First, in FTS observations (with the 30 -arc sec FOV Winston cone) at the CSO on 22 and 24 July 1995, spectra of Uranus (diameter $=3.74$ arc sec at the 1-bar level) were calibrated with respect to Mars (diameter $=5.20$ arc sec) as just outlined. The resultant spectrum of Mars on the first of these nights has already been displayed in Fig. 5(f), and the ratio of the two planetary antenna temperatures (averaged over the two nights), corrected for the solid-angle ratio (i.e., the intrinsic brightness temperature ratio for the median observation date), is shown in Fig. 6(a) at a spectral resolution of $7.2 \mathrm{GHz}$. Scaling by the same Rudy model for Mars as discussed in Subsection 5.A then provides the calibrated Uranus brightness temperature spectrum shown in Fig. 6(b). In like fashion, Neptune's spectrum was calibrated relative to that of Uranus on 19 September 1995 (when the diameters at the 1-bar level were 2.30 and 3.67 arc sec, respectively). The resultant solid-angle-cor-
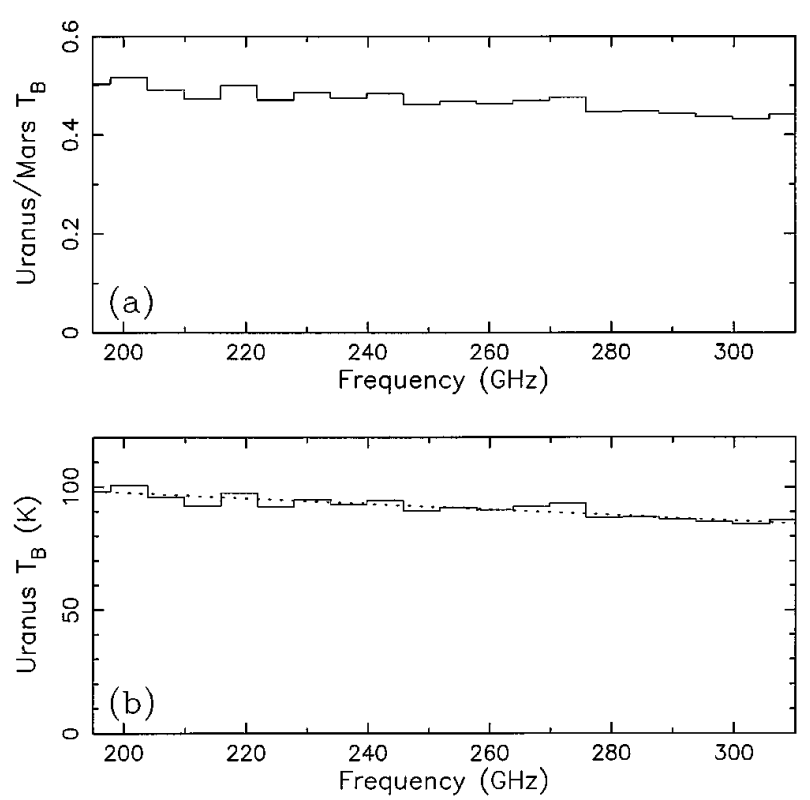

Fig. 6. (a) Observed brightness temperature ratio of Uranus and Mars (average of the 22 and 24 July 1995 observations). (b) The derived intrinsic brightness temperature spectrum of Uranus, assuming a Rudy ${ }^{33}$ brightness temperature model for Mars. Both panels are at a resolution of $7.2 \mathrm{GHz}$. 

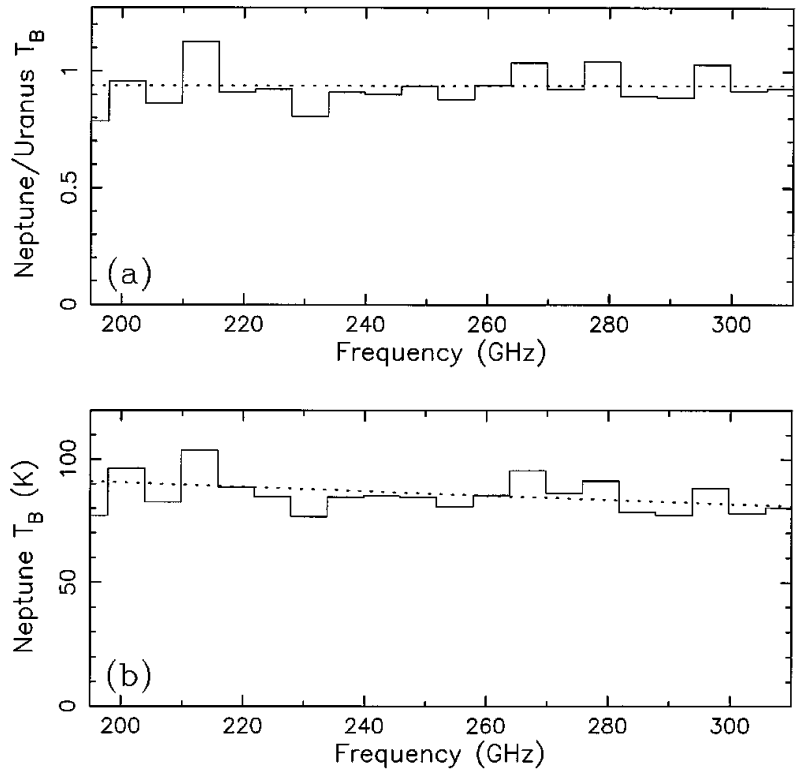

Fig. 7. (a) Observed brightness temperature ratio of Neptune and Uranus on 19 September 1995. (b) The derived intrinsic brightness temperature spectrum of Neptune, with the Uranus spectrum of Fig. 6(b) used to calibrate. Both panels are at a resolution of $7.2 \mathrm{GHz}$.

rected brightness temperature ratio is shown in Fig. $7(\mathrm{a})$, again at a spectral resolution of $7.2 \mathrm{GHz}$. Note that this intrinsic brightness temperature ratio is determined without reference to Mars. The ratio appears quite flat, and its best-fit constant value is 0.94 , identical to that determined with broadband filter observations. ${ }^{28}$ Using the previously calibrated Uranus spectrum of Fig. 6(b) to normalize the Neptune-to-Uranus ratio then yields the calibrated absolute brightness temperature spectrum of Neptune shown in Fig. 7(b).

Figures $6(\mathrm{~b})$ and $7(\mathrm{~b})$ represent the first measurements of continuous planetary absolute brightness temperature spectra at wavelengths beyond the far infrared; previously, only broadband filter (submillimeter and near millimeter) and single-frequency heterodyne (radio and microwave) measurements of planetary continuum levels were available. The measured brightness temperatures for Uranus and Neptune are both in very good agreement with measured broadband temperatures at these frequencies, ${ }^{28}$ but the FTS spectra immediately provide the temperature dependence across the band. From the best fits to the spectra in Figs. 6(b) and 7(b), it can be seen that both planets decrease regularly in temperature (by $\sim 10 \mathrm{~K}$ per $100 \mathrm{GHz}$ ) across the observed interval, because of the increasing importance of $\mathrm{H}_{2}$ opacity toward higher frequencies. ${ }^{34}$ The best linear temperature fits for the 195-310$\mathrm{GHz}$ range are

$$
\begin{array}{ll}
T_{U}=97.4-0.109\left(v_{\mathrm{GHz}}-200\right), & \text { (Uranus), } \\
T_{N}=90.7-0.090\left(v_{\mathrm{GHz}}-200\right), & \text { (Neptune). }
\end{array}
$$

The implications of these measured brightness tem- peratures for the constituents of jovian planet atmospheres will be discussed elsewhere.

\section{Summary and Outlook}

Given these initial successes, the use of FTS observations for the absolute calibration of near-millimeterand submillimeter-wavelength planetary brightness temperature spectra is established. With further observations and refinements to the beam modeling, it should then be possible to extend these measurements to yield calibrated continuum emission levels for all the planets across all the submillimeter-wave bands. In particular, it should be possible to calibrate Mars accurately relative to the Moon and then to use Mars as a calibrator for the rest of the planets. For Mars, this will both obviate the need to extrapolate data and models from distant wave bands and also provide better constraints on its surface dielectric properties. ${ }^{33}$ For the jovian planets, the continuum temperatures reflect the opacities of the atmospheric constituents. ${ }^{34,35}$ Of course, beyond the accurate determination of continuum levels, FTS's will likely be quite productive in searches for planetary absorption lines. ${ }^{10,13}$

The authors thank T. Encrenaz for assistance during the July observations. This work is supported by National Science Foundation grant AST9313929.

\section{References}

1. W. A. Traub and M. T. Stier, "Theoretical atmospheric transmission in the mid- and far-infrared at four altitudes," Appl. Opt. 15, 364-377 (1976).

2. E. Grossman, AT atmospheric transmission software, Airhead Software, Boulder, Colorado (1989).

3. J. E. Chamberlain, G. W. Chantry, F. D. Findlay, H. A. Gebbie, J. E. Gibbs, N. W. B. Stone, and A. J. Wright, "The spectral transmission at infra-red wavelengths of Michelson interferometers with dielectric film beam-dividers," Infrared Phys. 6, 195-203 (1966).

4. W. T. Welford and R. Winston, High Collection Nonimaging Optics (Academic, San Diego, 1989), Chap. 4.

5. T. G. Phillips, "Millimeter and submillimeterwave receivers," in Astronomy with Millimeter and Submillimeter Wave Interferometry, M. Ishiguro and W. J. Welch, eds. (ASP, San Francisco, 1994), pp. 68-77.

6. J. E. Carlstrom and J. Zmuidzinas, "Millimeter and submillimeter techniques," in Reviews of Radio Science 1993-1995, W. R. Stone, ed. (Oxford U. Press, Oxford, 1996).

7. W. D. Duncan, E. I. Robson, P. A. R. Ade, M. J. Griffin, and G. Sandell, "A millimetre/submillimetre common-user photometer for the James Clerk Maxwell Telescope," Mon. Not. R. Astron. Soc. 243, 126-132 (1990).

8. E. Kreysa, E. E. Haller, H. P. Gemünd, C. G. T. Haslam, R. Lemke, and A. W. Sievers, "First results from a small bolometer array on the IRAM 30-m telescope," in Proceedings of the Fourth International Symposium on Space Terahertz Technology (UCLA, Los Angeles, 1993), pp. 692-697.

9. C. L. Bennett, D. J. Fixsen, G. Hinshaw, J. C. Mather, S. H. Moseley, E. L. Wright, R. E. Eplee, Jr., J. Gales, T. Hewagama, R. B. Isaacman, R. A. Shafer, and K. Turpie, "Morphology of the interstellar cooling lines detected by COBE,"Astrophys. J. 434, 587-598 (1994).

10. E. W. Weisstein and E. Serabyn, "Detection of the $267 \mathrm{GHz}$ 
$J=1-0$ rotational transition of $\mathrm{PH}_{3}$ in Saturn with a new Fourier transform spectrometer," Icarus 109, 367-381 (1994).

11. E. Serabyn and E. W. Weisstein, "Fourier transform spectroscopy of the Orion molecular cloud core," Astrophys. J. 451, 238-251 (1995).

12. E. Serabyn, "Astronomical, atmospheric, and wavefront studies with a submillimeter wavelength interferometer," in Amazing Light: A Volume Dedicated to Charles Townes on His 80th Birthday, R. Y. Chiao, ed. (Springer, New York, 1995).

13. D. A. Naylor, T. A. Clark, and G. R. Davis, "A polarizing Fourier transform spectrometer for astronomical spectroscopy at submillimeter and mid-infrared wavelengths," in Instrumentation in Astronomy VIII, D. L. Crawford and E. R. Craine, eds., Proc. SPIE 2198, 703-714 (1994).

14. D. A. Naylor, G. R. Davis, M. J. Griffin, T. A. Clark, D. Gautier, and A. Marten, "Broad-band spectroscopic detection of $\mathrm{CO}$ $J=3-2$ tropospheric absorption in the atmosphere of Neptune," Astron. Astrophys. 291, L51-L53 (1994).

15. H. W. Schnopper and R. I. Thompson, "Fourier spectrometers," in Methods of Experimental Physics, M. L. Meeks, ed. (Academic, New York, 1974), Vol. 12A, pp. 491-529.

16. J. W. Brault, "Fourier transform spectroscopy," in High Resolution in Astronomy: 15th Advanced Course of the Swiss Society of Astronomy and Astrophysics, A. Benz, M. Huber, and M. Mayor, eds. (Geneva Observatory, Sauverny, Switzerland, 1985).

17. E. Serabyn, C. R. Masson, and T. G. Phillips, "Surface figure measurements of radio telescopes with a shearing interferometer,"Appl. Opt. 30, 1227-1241 (1991).

18. D. H. Martin, "Polarizing (Martin-Puplett) interferometric spectrometers for the near- and submillimeter spectra," in Infrared and Millimeter Waves (Academic Press, New York, 1982), Vol. 6, Chap. 2, pp. 65-148.

19. Emerson \& Cuming, Inc., Woburn, Mass.

20. J. D. Kraus, Radio Astronomy, 2nd ed. (Cygnus-Quasar, Powell, Ohio, 1986).

21. A. A. Penzias and C. A. Burrus, "Millimeter-wavelength radio-astronomy techniques," Ann. Rev. Astron. Astrophys. 11, 51-72 (1973).

22. B. L. Ulich and R. W. Haas, "Absolute calibration of millimeter- wavelength spectral lines," Astrophys. J. Supp. 30, 247-258 (1976).

23. M. L. Kutner and B. L. Ulich, "Recommendations for calibration of millimeter-wavelength spectral line data," Astrophys. J. 250, 341-348 (1981).

24. M. L. Kutner, L. Mundy, and R. J. Howard, "Interpretation of absolute line intensities on the NRAO 11 meter and other millimeter wave telescopes," Astrophys. J. 283, 890-894 (1984).

25. M. A. Gordon, J. W. M. Baars, and W. J. Cocke, "Observations of radio lines from unresolved sources: telescope coupling, Doppler effects, and cosmological corrections," Astron. Astrophys. 264, 337-344 (1992).

26. P. W. Rosenkranz, "Absorption of microwaves by atmospheric gases," in Atmospheric Remote Sensing by Microwave Radiometry, M. A. Janssen, ed. (Wiley, New York, 1994), Chap. 2.

27. B. L. Ulich, J. R. Dickel, and I. de Pater, "Planetary observations at a wavelength of $1.32 \mathrm{~mm}$," Icarus 60, 590-598 (1984).

28. M. J. Griffin and G. S. Orton, "The near-millimeter brightness temperature spectra of Uranus and Neptune," Icarus 105, 537-547 (1993).

29. G. Heiken, D. Vaniman, and B. M. French, Lunar Sourcebook (Cambridge U. Press, Cambridge, 1991), p. 34.

30. J. L. Linsky, "The Moon as a proposed radiometric standard for microwave and infrared observations of extended sources," Astrophys. J. Supp. 25, 163-204 (1973).

31. J. Ruze, "Antenna tolerance theory-a review," Proc. IEEE 54, 633-640 (1966).

32. M. Born and E. Wolf, Principles of Optics, 6th ed. (Pergamon, Oxford, 1980), pp. 463-464.

33. D. J. Rudy, "Mars: high resolution VLA observations at wavelengths of 2 and $6 \mathrm{~cm}$ and derived properties," Ph.D. dissertation (California Institute of Technology, Pasadena, Calif., 1987).

34. P. Dore, L. Nencini, and G. Birnbaum, "Far infrared absorption in normal $\mathrm{H}_{2}$ from 77 to $298 \mathrm{~K}$," J. Quant. Spectrosc. Radiat. Transfer 30, 245-253 (1983).

35. I. de Pater and S. Massie, "Models of the millimetercentimeter spectra of the giant planets," Icarus 62, 143-171 (1985). 